ARTICLE

Received 15 Jan 2014 | Accepted 4 Jun 2014 | Published 7 Jul $2014 \quad$ DOl: 10.1038/ncomms5291

\title{
Suppression of the critical thickness threshold for conductivity at the $\mathrm{LaAlO}_{3} / \mathrm{SrTiO}_{3}$ interface
}

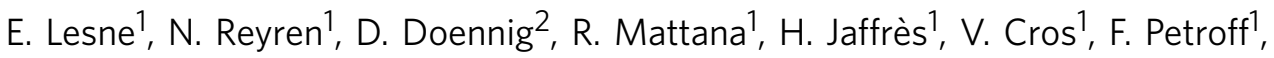 \\ F. Choueikani ${ }^{3}$, P. Ohresser ${ }^{3}$, R. Pentcheva ${ }^{2,4}$, A. Barthélémy ${ }^{1} \&$ M. Bibes ${ }^{1}$
}

Perovskite materials engineered in epitaxial heterostructures have been intensely investigated during the last decade. The interface formed by an $\mathrm{LaAlO}_{3}$ thin film grown on top of a $\mathrm{TiO}_{2}$-terminated $\mathrm{SrTiO}_{3}$ substrate hosts a two-dimensional electronic system and has become the prototypical example of this field. Although controversy exists regarding some of its physical properties and their precise origin, it is universally found that conductivity only appears beyond an $\mathrm{LaAlO}_{3}$ thickness threshold of four unit cells. Here, we experimentally demonstrate that this critical thickness can be reduced to just one unit cell when a metallic film of cobalt is deposited on top of $\mathrm{LaAlO}_{3}$. First-principles calculations indicate that $\mathrm{Co}$ modifies the electrostatic boundary conditions and induces a charge transfer towards the $\mathrm{Ti}$ $3 d$ bands, supporting the electrostatic origin of the electronic system at the $\mathrm{LaAlO}_{3} / \mathrm{SrTiO}_{3}$ interface. Our results expand the interest of this low-dimensional oxide system from in-plane to perpendicular transport and to the exploration of elastic and inelastic tunnel-type transport of (spin-polarized) carriers.

\footnotetext{
${ }^{1}$ Unité Mixte de Physique CNRS/Thales, 1 Avenue A. Fresnel, 91767 Palaiseau, France and Université Paris-Sud, 91405 Orsay, France. ${ }^{2}$ Department of Earth and Environmental Sciences, Section Crystallography and Center of Nanoscience (CENS), University of Munich, Theresienstrasse 41, DE-80333 Munich, Germany. ${ }^{3}$ Synchrotron SOLEIL, L'Orme des Merisiers, Saint-Aubin, BP 48, 91192 Gif-sur-Yvette, France. ${ }^{4}$ Faculty of Physics, University of Duisburg-Essen, D-47048 Duisburg, Germany. Correspondence and requests for materials should be addressed to E.L. (email: edouard.lesne@thalesgroup.com) or to M.B. (email: manuel.bibes@thalesgroup.com).
} 
C ombined in heterostructures, oxide thin films offer a new playground with virtually unlimited combinations to realize artificial systems and to explore emerging phenomena absent in bulk compounds ${ }^{1}$. Ohtomo and Hwang ${ }^{2}$ striking discovery of conducting $\mathrm{LaAlO}_{3} / \mathrm{SrTiO}_{3}$ heterointerfaces has fast-tracked this system as the fruit fly for such investigations. The $\mathrm{LaAlO}_{3} / \mathrm{SrTiO}_{3}$ system exhibits gate-tunable quasi-twodimensional electronic conduction ${ }^{3}$, Rashba spin-orbit interaction $^{4,5}$ and superconductivity ${ }^{6-8}$, displays indications of magnetism $\mathrm{m}^{9,10}$ and even close coexistence of the latter two ${ }^{11-13}$. This makes the $\mathrm{LaAlO}_{3} / \mathrm{SrTiO}_{3}$ system attractive for fundamental studies as well as promising for potential applications such as oxide-based electronics ${ }^{14-16}$.

While the perovskite crystals $\mathrm{LaAlO}_{3}(\mathrm{LAO})$ and $\mathrm{SrTiO}_{3}(\mathrm{STO})$ are both wide band-gap insulators, the growth of an epitaxial LAO film on a $\mathrm{TiO}_{2}$-terminated, (001)-oriented STO substrate leads to an insulator-to-metal transition when the LAO thickness reaches 4 unit cells $(\mathrm{uc})^{3}$. Owing to their ionic nature, the $\mathrm{STO}(001)$ substrate (non-polar) and the $\mathrm{LAO}(001)$ film (polar) form a strongly polar interface. This results in an electrostatic potential that increases as the LAO thickness is increased, leading to an electronic reconstruction with charge transferred towards the STO top- $-\mathrm{TiO}_{2}$ subplane ${ }^{17}$.

Recently, first-principles calculations predicted a conductive system at the LAO/STO interface for heterostructures with various metallic capping layers, and an LAO thickness of $2 \mathrm{uc}^{18}$. In this letter, we present results of electrical magnetotransport and X-ray absorption spectroscopy (XAS) experiments on LAO/ STO samples with either bare LAO surfaces or a metallic capping layer of cobalt, combined with density functional theory (DFT) calculations. We demonstrate the existence of metallic conductivity on the STO side for an LAO thickness as thin as 1 uc.

\section{Results}

Magnetotransport measurements of Co/LAO/STO samples. LAO films were grown by pulsed laser deposition (PLD) on $\mathrm{TiO}_{2}$-terminated STO(001) substrates and followed by in situ magnetron sputtering deposition of a $2-2.5-\mathrm{nm}$ cobalt thin film, capped with a 3-nm $\mathrm{AlO}_{x}$ layer to prevent Co oxidation. Details concerning samples fabrication and characterization can be found in the Methods section and in the Supplementary Information (Supplementary Note 1).

The unpatterned samples were electrically contacted with aluminium wires by ultrasonic wedge bonding. Four-point transport measurements (in-plane longitudinal $R_{\mathrm{xx}}$ and transverse $R_{\mathrm{xy}}$ magnetoresistances) were performed by connecting the corners of the square sample in the van der Pauw geometry. Figure la shows the measured in-plane longitudinal resistance $R_{\mathrm{xx}}^{\text {meas }}$ versus temperature of Co/LAO/STO heterostructures with various LAO thicknesses, and of a Co/STO control sample for which the STO substrate experienced the same procedure of thermal and oxygen pressure cycles as for the LAO/STO samples. While the Co/STO sample shows very little variation of its resistance $(<1 \%)$ across the explored temperature range, the Co-capped LAO/STO samples display a strong reduction in their resistance as temperature is decreased, which readily suggests that the LAO/STO interface conducts, even for samples with an LAO film thickness smaller than $4 \mathrm{uc}$.

Following these observations, we now model the $\mathrm{Co} / \mathrm{LAO}(n \geq 1 \mathrm{uc}) / \mathrm{STO}$ heterostructures by two conducting layers in parallel composed of the Co overlayer and the STO sublayer. According to this simple picture, and supposing a temperatureindependent resistance of the Co capping layer (see Fig. 1a; Supplementary Fig. 1), the temperature evolution of the STO sublayer's longitudinal resistance is given by:

$$
R_{\mathrm{xx}}^{\mathrm{STO}}(T)=\frac{R_{\mathrm{xx}}^{\mathrm{Co}} \cdot R_{\mathrm{xx}}^{\text {meas }}(T)}{R_{\mathrm{xx}}^{\mathrm{Co}}-R_{\mathrm{xx}}^{\text {meas }}(T)} .
$$

The sheet resistance of the STO sublayer is defined as $R_{\square}^{\mathrm{STO}}=R_{\mathrm{xx}}^{\mathrm{STO}}(T) \times \frac{\pi}{\ln (2)}$, assuming a homogeneous conductance in the geometry of the inset of Fig. $1 \mathrm{a}^{19}$, supported by the fact that $R_{\mathrm{xx}} \approx R_{\mathrm{yy}}$ within a few percent. For each sample, we calculate $R_{\mathrm{xx}}^{\mathrm{STO}}(T)$ by taking the data set as $R_{\mathrm{xx}}^{\text {meas }}(T)$, and $R_{\mathrm{xx}}^{\mathrm{Co}}$ as the measured value at room temperature $\left(R_{\mathrm{xx}}^{\mathrm{Co}}=R_{\mathrm{xx}}^{\text {meas }}(290 \mathrm{~K})\right)$ for the corresponding sample. Figure $1 \mathrm{~b}$ displays the calculated sheet resistance $R_{\square}^{\text {STO }}$ of the $\mathrm{Co} / \mathrm{LAO} / \mathrm{STO}$ samples in the lowtemperature range (below $100 \mathrm{~K}$ ). a

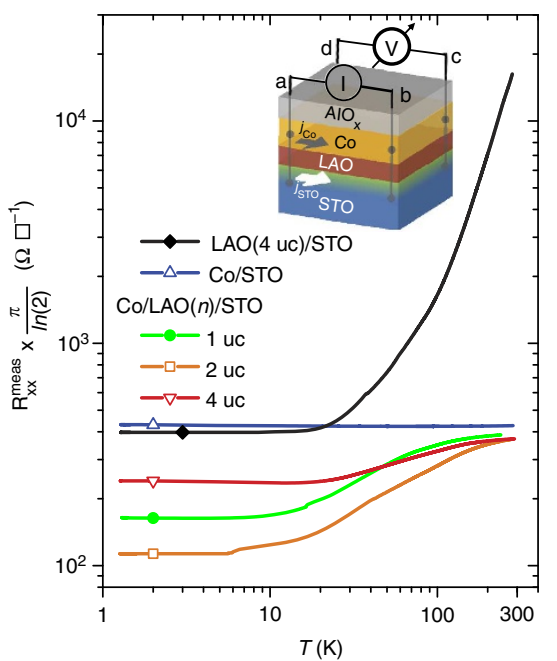

b

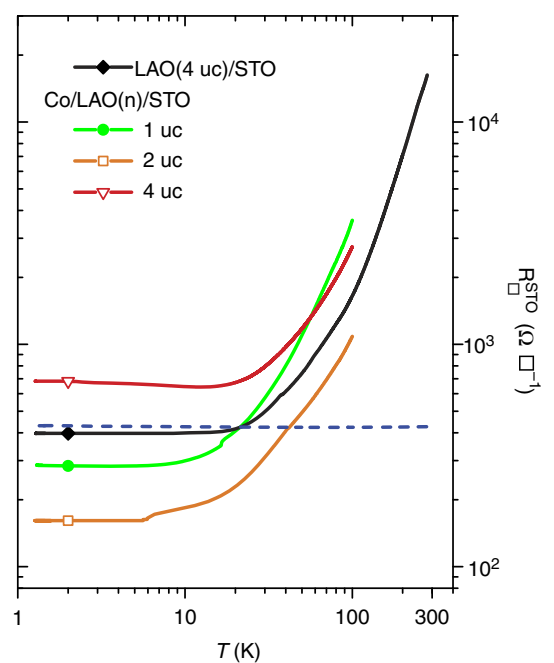

Figure 1 | Sheet resistances as a function of temperature. (a) Measured longitudinal resistance $R_{\mathrm{xx}}^{\text {meas }}$ versus temperature $T$ of LAO(4 uc)/STO and $\mathrm{Co} / \mathrm{LAO}(n) / \mathrm{STO}$ samples showing a clear metallic behaviour. The Co/STO resistance remains almost unchanged from 300 to $1.4 \mathrm{~K}(\Delta R / R<1 \%)$. Inset: measurement configuration. The thick arrows in the sketch represent the current flowing in the Co layer and in the STO. (b) Calculated sheet resistance $R_{\square}^{\text {STO }}$ of the conducting STO sublayer in Co/LAO(n)/STO samples (according to equation (1)), and measured sheet resistance of the LAO(4 uc)/STO samples. The blue dotted line corresponds to the Co/STO data set. 
a

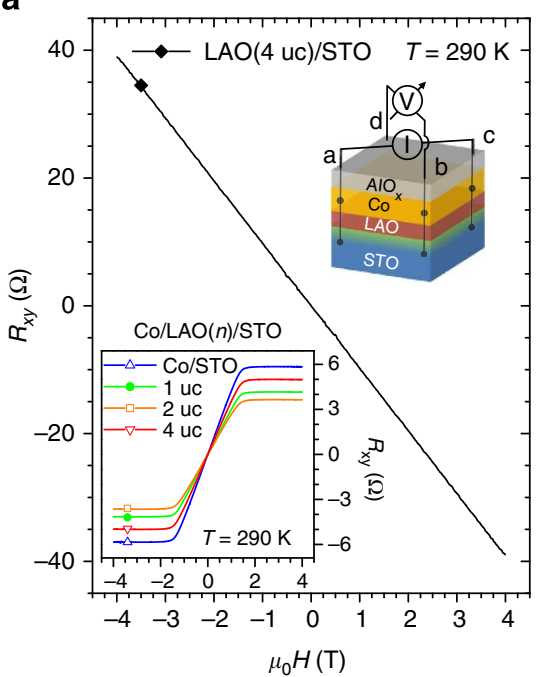

b

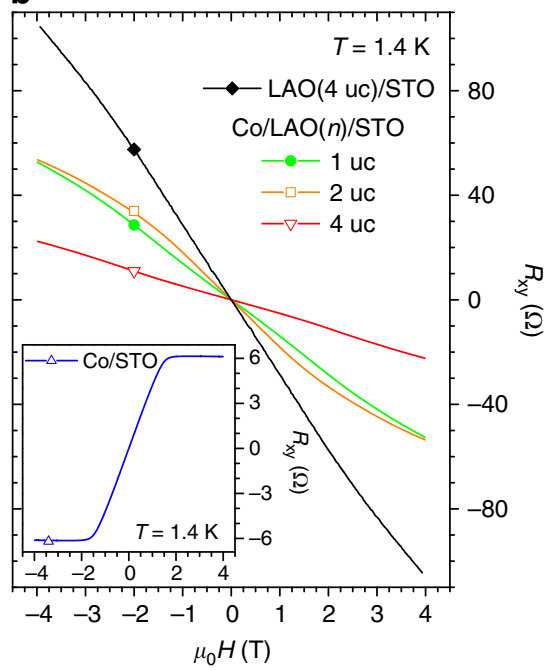

Figure 2 | Hall effect measurements. (a) Antisymmetrized Hall resistance $R_{x y}$ as a function of applied magnetic field $\mu_{0} H$ of an LAO(4 uc)/STO sample displaying a linear Hall effect at room temperature. Inset: $R_{x y}$ versus $\mu_{0} H$ for various Co/LAO(n)/STO samples at $290 \mathrm{~K}$ showing cobalt dominated AHE at low magnetic field. (b) Antisymmetrized Hall resistance $R_{x y}$ versus $\mu_{0} H$ of an LAO(4uc)/STO sample and of Co/LAO(n)/STO at $1.4 \mathrm{~K}$. Inset: $R_{\mathrm{xy}}$ versus $\mu_{0} H$ for Co/STO at $1.4 \mathrm{~K}$ similar to the one observed at $290 \mathrm{~K}$.

Next, we focus on transport experiments carried out in Hall configuration at room temperature and at low temperature $(1.4 \mathrm{~K})$. The measured transverse magnetoresistance curves were antisymmetrized $\left(R_{\mathrm{xy}}(H)=\left[R_{\mathrm{xy}}^{\text {meas }}(H)-R_{\mathrm{xy}}^{\text {meas }}(-H)\right] / 2\right)$. Antisymmetrized traces $R_{\mathrm{ac}-\mathrm{db}}(H)$ and $R_{\mathrm{db}-\mathrm{ca}}(H)$ appear indistinguishable in Fig. 2a,b, suggesting again good sample homogeneity and no artefacts due to the data analysis. In the case of the $\mathrm{LAO}(4 \mathrm{uc}) / \mathrm{STO}$ sample, Hall measurements taken at room temperature show a linear behaviour for magnetic field up to $4 \mathrm{~T}$ (see Fig. 2a) with an extracted sheet carrier densitiy $n_{\square}=6.4$ $\times 10^{13} \mathrm{~cm}^{-2}$. In contrast, $\mathrm{Co} / \mathrm{STO}$ and $\mathrm{Co} / \mathrm{LAO} / \mathrm{STO}$ samples exhibit at room temperature a Hall effect dominated by the cobalt layer, independent of the LAO thickness (see inset of Fig. 2a), with a low-magnetic-field regime $\left(\left|\mu_{0} H\right| \leq 1.2 \mathrm{~T}\right)$ displaying the anomalous Hall effect characteristic of a ferromagnetic metal. In the high-field regime $\left(\left|\mu_{0} H\right| \geq 2.2 \mathrm{~T}\right)$, the Co magnetization is rotated out of plane and the Co-capped samples exhibit a normal Hall effect with a weak negative slope of order $-10 \mathrm{~m} \Omega \mathrm{T}^{-1}$ (that is a carrier density of order $10^{23} \mathrm{~cm}^{-3}$ ).

Figure $2 \mathrm{~b}$ displays Hall data acquired at $1.4 \mathrm{~K} . R_{\mathrm{xy}}(H)$ measured at $1.4 \mathrm{~K}$ in $\mathrm{Co} / \mathrm{STO}$ shows essentially no change when compared with room temperature measurements (see insets of Fig. 2a,b). On the other hand, $R_{\mathrm{xy}}(H)$ behaviour of $\mathrm{Co} /$ $\mathrm{LAO}(n=1,2,4 \mathrm{uc}) / \mathrm{STO}$ samples at $1.4 \mathrm{~K}$ display striking similarities with those carried out on uncapped $\mathrm{LAO}(4 \mathrm{uc}) / \mathrm{STO}$ samples. The Hall resistance trace of the $\mathrm{LAO}(4 \mathrm{uc}) / \mathrm{STO}$ sample is not purely linear at $1.4 \mathrm{~K}$, a behaviour believed to arise from the multi-band structure of $\mathrm{STO}^{20-22}$. Evidence for this is given from the DFT calculations in the Supplementary Information (see Supplementary Fig. 2). We only consider the 'high field' regime $\left(\left|\mu_{0} H\right| \geq 3 \mathrm{~T}\right)$ where $n_{\square}$, the total electron density in the STO, is given by $n_{\square}=-\frac{1}{|e| \cdot R_{H}}$, with $e$ the elementary charge and $R_{H}$ the Hall coefficient determined for $\left|\mu_{0} H\right| \geq 3 \mathrm{~T}$. The kink observed in the $\mathrm{LAO}(4 \mathrm{uc}) / \mathrm{STO}$ Hall trace $R_{\mathrm{xy}}(H)$ is well reproduced in Cocapped LAO samples at $1.4 \mathrm{~K}$, regardless of the LAO thickness. This close resemblance for both sets of samples suggests a common host for the observed electronic transport. The measured Hall coefficients, and related carrier densities show no correlation with LAO thickness. Taking the sheet resistance and Hall coefficient at $1.4 \mathrm{~K}$, one can estimate the electronic mobility $\mu_{\mathrm{H}}^{\mathrm{STO}}=R_{\mathrm{H}} / R_{\square}^{\mathrm{STO}}$ of the STO sublayer in Co/LAO $/ \mathrm{STO}$ samples. Values in the range of $\left(1-6 \times 10^{2} \mathrm{~cm}^{2} \mathrm{~V}^{-1} \mathrm{~s}^{-1}\right)$ are found. We note that the very thin Co layer is not expected to play a role in Hall effect measurements at $1.4 \mathrm{~K}$, owing to its much lower mobility $\left(\mu_{\mathrm{H}}^{\mathrm{Co}}<1 \mathrm{~cm}^{2} \mathrm{~V}^{-1} \mathrm{~s}^{-1}\right.$ determined experimentally), thus supporting our simple analysis.

Hence, our transport data collected on Co/LAO/STO heterostructures clearly indicate the presence of an electronic system at the LAO/STO interface whose behaviour is very similar to the usual $\mathrm{LAO}(n \geq 4 \mathrm{uc}) / \mathrm{STO}$, even for LAO as thin as a single unit cell. Furthermore, the estimated carrier densities (several $10^{13}$ $\times 10^{14} \mathrm{~cm}^{-2}$ ) in Co/LAO/STO samples are comparable to values typically found for quasi-two-dimensional electronic systems at LAO/STO interfaces $3,23,24$.

X-ray absorption spectroscopy experiments. To gain further insight into the electronic structure of $\mathrm{Co} / \mathrm{LAO} / \mathrm{STO}$ systems, we have performed XAS measurements at the DEIMOS beamline of Synchrotron SOLEIL ${ }^{25}$. XAS probes transition from core electronic states to excited electronic states. The energy of the incident X-ray beam can be tuned to the Ti $L_{2,3}$ absorption edge to probe electronic transitions from $2 p_{3 / 2}$ and $2 p_{1 / 2}$ to $3 d$ levels (see Fig. $3 a)^{26}$. Therefore, the preferential occupancy of states with different orbital symmetries can be probed by X-ray linear dichroism (XLD), which is the difference in XAS measured with a linear horizontal $(\mathrm{LH})$ or vertical $(\mathrm{LV})$ polarization of the X-ray beam (see Fig. 3b], that is, $\mathrm{XLD}=(\mathrm{LH}-\mathrm{LV})$. Conversely, the isotropic signal is defined as the mean of the two: $\mathrm{ISO}=(\mathrm{LH}+\mathrm{LV}) / 2$.

The XAS experiments were performed at the Ti $L_{2,3}$ absorption edge, on the same $\mathrm{Co} / \mathrm{STO}$ and $\mathrm{Co} / \mathrm{LAO}(2 \mathrm{uc}) / \mathrm{STO}$ samples previously shown in Figs 1 and 2, as well as on a bare STO substrate, on an insulating $\mathrm{LAO}(2 \mathrm{uc}) / \mathrm{STO}$ heterostructure and on a conducting $\mathrm{LAO}(4 \mathrm{uc}) / \mathrm{STO}$ sample. All spectra were collected at $300 \mathrm{~K}$ in total electron yield mode in order to be surface sensitive. In Fig. 3, the ISO signals appear to be largely of $\mathrm{Ti}^{4+}$ character for all samples ${ }^{27}$. The XLD signals, however, display significant differences between the samples (see Fig. 3d). In LAO/STO samples with 2 and 4 uc of LAO, the XLD shows a sign reversal with respect to that obtained from a bare STO substrate. This is related to an inverted (negative versus positive) 
a

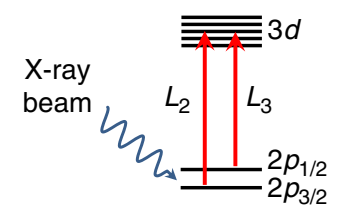

f

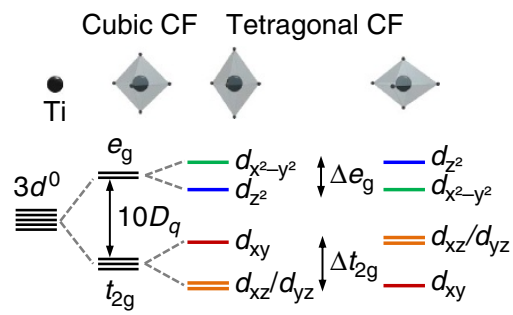

[A] b

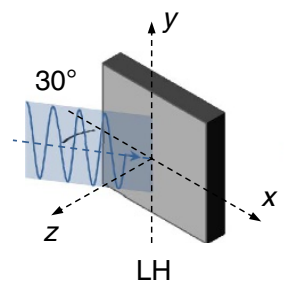

e
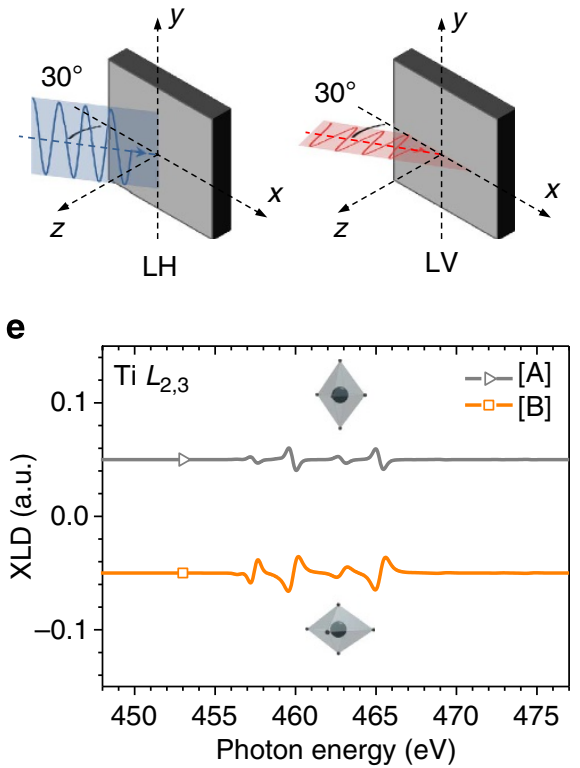

LV
C

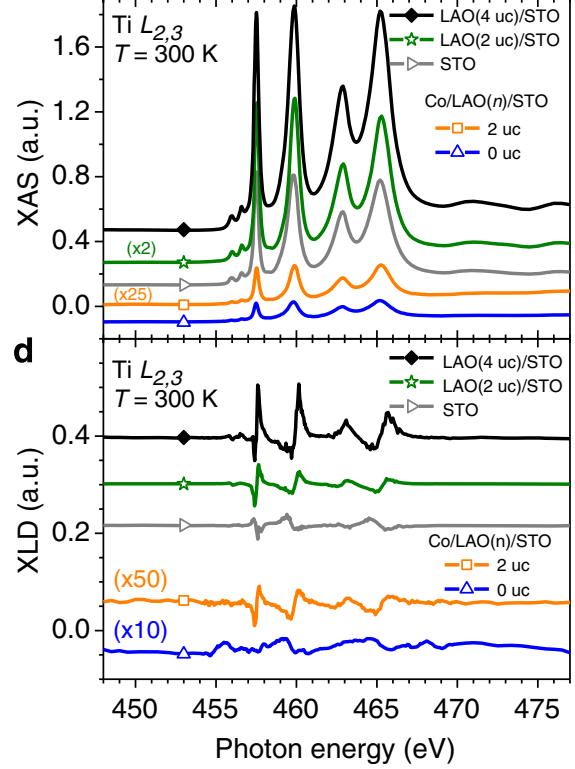

Figure 3 | X-ray absorption spectroscopy at Ti $\boldsymbol{L}$ edge. (a) Sketch of the $\mathbf{X}$-ray absorption spectroscopy (XAS) process depicting the $2 p \rightarrow 3 d$ electronic transitions at the Ti $L_{2,3}$ edge. (b) Scheme of the XAS measurement geometries with linearly horizontal (LH) and linearly vertical (LV) polarized light. The incident beam forms a $30^{\circ}$ angle with the sample surface $(x, y)$. (c) Experimentally measured isotropic signals (ISO) versus photon energy of a $\mathrm{STO}(001)$ substrate, and of various (Co/)LAO(n)/STO samples. Spectra acquired at $300 \mathrm{~K}$ in total electron yield mode. (d) The corresponding experimental X-ray linear dichroism (XLD) signals show a sign inversion between the XLD of STO and Co/STO and that of LAO(2,4 uc) and Co/LAO(2 uc) samples. (e) Calculated XLD that reproduces the experimental XLD of the STO bare substrate (circle, [A]) and of the Co/LAO(2 uc)/STO sample (square, [B]). (f) The cubic crystal field (CF) splits the $\mathrm{Ti}^{4+} 3 d$ states into $e_{\mathrm{g}}$ and $t_{2 \mathrm{~g}}$ levels. A tetragonal CF further lifts the orbital degeneracy, with: $[\mathrm{A}]$ case of STO, [B] case of (Co/)LAO/STO.

a

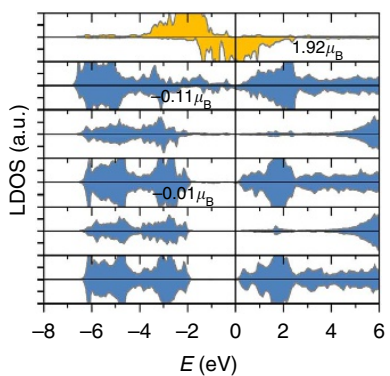

b

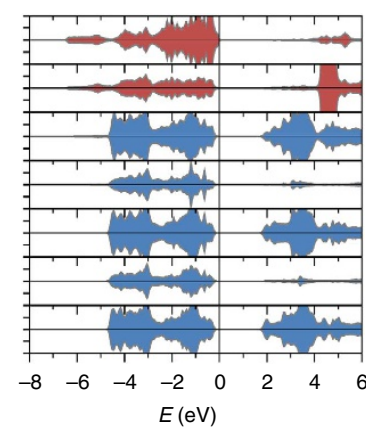

C

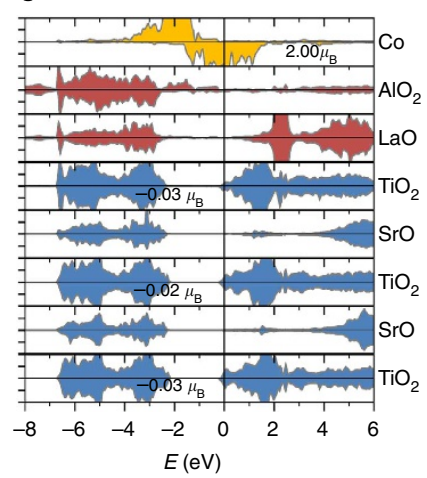

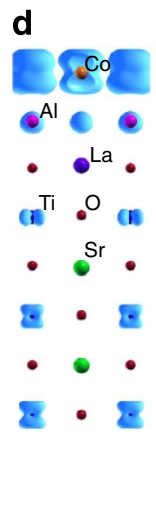

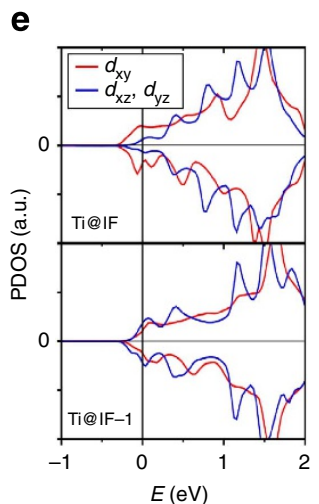

Figure 4 | Layer-resolved density of states. LDOS of (a) Co(1 ML)/STO, (b) LAO(1 uc)/STO and (c) Co(1 ML)/LAO(1 uc)/STO heterostructures within GGA with a 4.5-uc-thick STO substrate. (d) Side view of $\mathrm{Co}(1 \mathrm{ML}) / \mathrm{LAO}(1 \mathrm{uc}) / \mathrm{STO}$ with the electron density integrated in the interval $E_{\mathrm{F}}-0.50 \mathrm{eV}$ to $E_{\mathrm{F}}$ giving insight into the Ti $3 d$ orbital occupation, where $E=E_{\mathrm{F}}=0$ corresponds to the Fermi level. (e) Projected density of states (PDOS) of $\mathrm{Ti}$ in the interface (IF) and IF-1 layer of $\mathrm{Co} / \mathrm{LAO}(1 \mathrm{uc}) / \mathrm{STO}$. Positive (negative) values correspond to majority (minority) spin.

splitting of the $3 d$ bands (see Fig. 3f). Interestingly, the XLD measured on the $\mathrm{Co} / \mathrm{LAO}(2 \mathrm{uc}) / \mathrm{STO}$ sample is also very different from that of the STO substrate and appears very similar to the uncapped LAO/STO samples. Although much noisier, the XLD signal of the Co/STO sample resembles that of the bare STO.

In bulk cubic STO, the crystal field splits the fivefold degenerate Ti $3 d$ bands into twofold $e_{\mathrm{g}}\left(d_{\mathrm{x}^{2}-\mathrm{y}^{2}}, d_{\mathrm{z}^{2}}\right)$ and threefold $t_{2 \mathrm{~g}}\left(d_{\mathrm{xy}}, d_{\mathrm{xz}}, d_{\mathrm{yz}}\right)$ degenerate bands 28 . In an attempt to clarify this band picture, we have performed atomic multiplet calculations using the CTM4XAS program ${ }^{29}$. It allowed us to explore the effect of a symmetry reduction of the system (for example, towards tetragonal), which distorts the $\mathrm{TiO}_{6}$ octahedra and lifts the degeneracy of the $e_{\mathrm{g}}$ and $t_{2 \mathrm{~g}}$ bands (see Fig. 3e,f). The measured XLD were well reproduced by using values of the energy splittings identical to those reported in the literature ${ }^{30,31}$. In the case of STO, we considered positive energy splittings $\Delta e_{\mathrm{g}}=40 \mathrm{meV}$ and $\Delta t_{2 \mathrm{~g}}=25 \mathrm{meV}^{30}$. In contrast, for the LAO/STO and Co/LAO/STO systems, negative energy splittings $\Delta e_{\mathrm{g}}=-100 \mathrm{meV}$ and $\Delta t_{2 \mathrm{~g}}=-50 \mathrm{meV}$ were considered ${ }^{31}$. We note that introducing a Co capping layer does not alter the fine structure of the XLD observed in uncovered LAO/STO samples. Interestingly, we have shown that Co-capped and uncapped LAO/STO systems have a similar subband structure, with a preferential $d_{\mathrm{xy}}$ orbital occupancy.

Incidentally, we performed X-ray magnetic circular dichroism experiments at the Ti $L_{2,3}$ edge on $\mathrm{Co} / \mathrm{LAO}(n) / \mathrm{STO}$ samples with $n=2$ and 4 uc samples (see Supplementary Fig. 3 ). The signal-tonoise ratio of our experiments allows us to estimate the $\mathrm{Ti}$ 
magnetic moment averaged over the total probing depth to be of the order of $10^{-2} \mu_{\mathrm{B}}$ per atom (Supplementary Note 2). This value is in agreement with those obtained from the DFT calculations presented below as well as from neutron reflectometry on LAO/STO superlattices ${ }^{32}$ and recent X-ray magnetic circular dichroism experiments on similarly prepared LAO/STOconducting interfaces ${ }^{33}$.

First-principles calculations. To elucidate the origin of the observed onset of conductivity below $4 \mathrm{uc}$ of LAO, DFT calculations were performed on $\mathrm{Co}(m) / \mathrm{LAO}(n) / \mathrm{STO}(001)$ (see Fig. 4) with the all-electron full-potential linearized augmented plane wave, as implemented in the WIEN2k $\operatorname{code}^{34,35}$. For the exchange-correlation functional, we used the generalized gradient approximation (GGA) ${ }^{36}$. The influence of electronic correlations beyond GGA is discussed in the Supplementary Note 3 (see also Supplementary Fig. 4). The in-plane lattice parameter is set to the GGA equilibrium lattice constant of STO $\left(a_{\mathrm{STO}}=3.92 \AA\right)$ and the atomic positions are fully relaxed within tetragonal symmetry. More details are provided in ref. 18 (see also the Methods section and Supplementary Note 3). Figure 4a shows the layer-resolved density of states of a (001)oriented STO substrate (4.5-uc thick) covered with one monolayer (ML) of Co. In contrast to the uncovered STO(001) substrate (not shown here), where the Fermi level is pinned at the top of the valence band defined by surface states at the topmost $\mathrm{TiO}_{2}$ layer, for $\mathrm{Co} / \mathrm{STO}(001)$ the Fermi energy $E_{\mathrm{F}}$ shifts to the bottom of the $\mathrm{Ti}$ conduction band, leaving the $\mathrm{Ti} 3 d$ band empty.

A similar change is observed when capping LAO/STO(001) with Co: In the uncovered $\mathrm{LAO}(1 \mathrm{uc}) / \mathrm{STO}$ system, the Fermi level lies at the top of the valence band (see Fig. 4b), determined by $\mathrm{O} 2 p$ states of the surface $\mathrm{AlO}_{2}$ layer, while the conduction band minimum is $2 \mathrm{eV}$ above the Fermi energy and is determined by Ti $3 d$ states. Adding a Co capping layer shifts the Fermi level to the bottom of the conduction band in $\mathrm{Co} / \mathrm{LAO}(1 \mathrm{uc}) / \mathrm{STO}$ (see Fig. 4c). In contrast to $\mathrm{LAO}(1 \mathrm{uc}) / \mathrm{STO}$ and $\mathrm{Co} / \mathrm{STO}$, in $\mathrm{Co} / \mathrm{LAO}(1 \mathrm{uc}) / \mathrm{STO}$ the Ti $3 d$ band is now partially occupied, with a small spin polarization of -0.02 to $-0.03 \mu_{B}$ per Ti. Hence a metallic bilayer is formed in $\mathrm{Co} / \mathrm{LAO} / \mathrm{STO}$, with a conducting surface (Co) and a conducting burried interface (STO side), consistent with the transport data. Furthermore, the $\mathrm{Ti}$ $3 d$ band occupation increases weakly with LAO thickness (see Supplementary Figs 5 and 6). Adding the Co capping layer

a

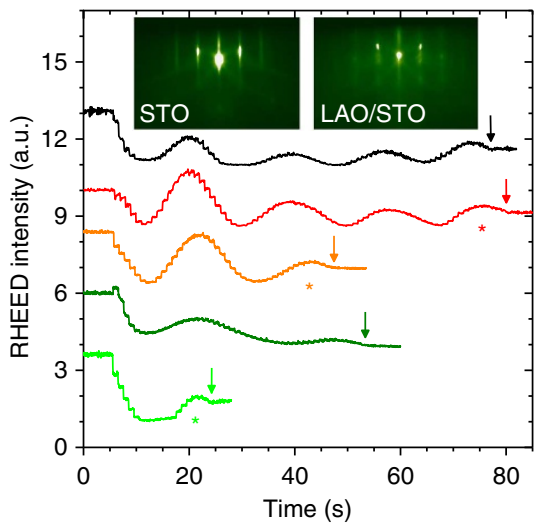

(largely) cancels the internal potential buildup within the polar LAO film. As found previously ${ }^{18}$, the position of the Fermi level with respect to the conduction band minimum in STO is determined by the work function of the metallic contact $(4.72 \mathrm{eV}$ for $1 \mathrm{ML} \mathrm{Co}$ and $4.28 \mathrm{eV}$ for $3 \mathrm{ML} \mathrm{Co}$ ). This, together with the still persisting polar discontinuity at the LAO/STO interface leads to a finite occupation of the $\mathrm{Ti} 3 d$ band at the interface.

Next, we explore the preferential orbital occupancy of the electronic system. The electron density of $\mathrm{Co} / \mathrm{LAO}(1 \mathrm{uc}) / \mathrm{STO}$, integrated between $E_{\mathrm{F}}-0.50 \mathrm{eV}$ and $E_{\mathrm{F}}$ reveals predominant $d_{\mathrm{xy}}$ orbital polarization of $\mathrm{Ti}$ in the interface layer and $d_{\mathrm{xz}} / d_{\mathrm{yz}}$ character in deeper layers (see Fig. $4 \mathrm{~d}$ ), which can also be seen on the projected density of states in Fig. 4e. The orbital polarization is consistent with the structural distortions of the octahedra within the $\mathrm{Co} / \mathrm{LAO}(1 \mathrm{uc}) / \mathrm{STO}$ heterostructure: We find that the average distance between the apical oxygens on both sides of Ti in the [001] direction is $3.92 \AA$ for the interfacial (IF) and $3.99 \AA$ for the IF-1 layers, respectively. In line with the experimentally determined $t_{2 \mathrm{~g}}$ splittings (Fig. $3 \mathrm{~d}-\mathrm{f}$ ), we find that a shortened (expanded) $\mathrm{TiO}_{6}$ octahedra average size in the [001] direction corresponds to $d_{\mathrm{xy}}$ orbitals lying lower (higher) in energy.

\section{Discussion}

Using four-point transport experiments, we have revealed the presence of metallic conduction at the $\mathrm{LaAlO}_{3} / \mathrm{SrTiO}_{3}$ interface for $\mathrm{Co} / \mathrm{LaAlO}_{3}(n) / \mathrm{SrTiO}_{3}$ heterostructures with $\mathrm{LaAlO}_{3}$ thicknesses $1 \mathrm{uc} \leq n \leq 4 \mathrm{uc}$. The linear dichroism observed through XAS evidences a compressive tetragonal distortion of the $\mathrm{TiO}_{6}$ octahedra for cobalt-capped and uncapped $\mathrm{LaAlO}_{3} / \mathrm{SrTiO}_{3}$ systems. Making use of atomic multiplet calculations, we found that in $\mathrm{Co} / \mathrm{LaAlO}_{3} / \mathrm{SrTiO}_{3}$ the $\mathrm{Ti}$ orbitals reconstruct from the situation in bare $\mathrm{SrTiO}_{3}$ substrates. As a result, the lowest energy level has a $d_{\mathrm{xy}}$ orbital character for $\mathrm{Co} / \mathrm{LaAlO}_{3} / \mathrm{SrTiO}_{3}$ systems, compared with $d_{\mathrm{xz}} / d_{\mathrm{yz}}$ for the bare $\mathrm{SrTiO}_{3}$. DFT calculations support this picture and predict structural distortions that agree with those inferred from XAS. Further, they indicate a finite occupancy of the $\mathrm{Ti} 3 d$ bands at the Fermi energy for the $\mathrm{Co} / \mathrm{LaAlO}_{3}(1 \mathrm{uc}) / \mathrm{SrTiO}_{3}$ heterostructure, with a predominant $d_{\mathrm{xy}}$ orbital character within the interfacial $\mathrm{TiO}_{2}$ layer. The metallic Co overlayer thus appears to modify the electrostatic boundary conditions of the LAO/STO system, leading to the occupation of the $\mathrm{Ti} 3 d$ bands. These observations support the electrostatic origin of the quasi-two-dimensional electronic systems at the

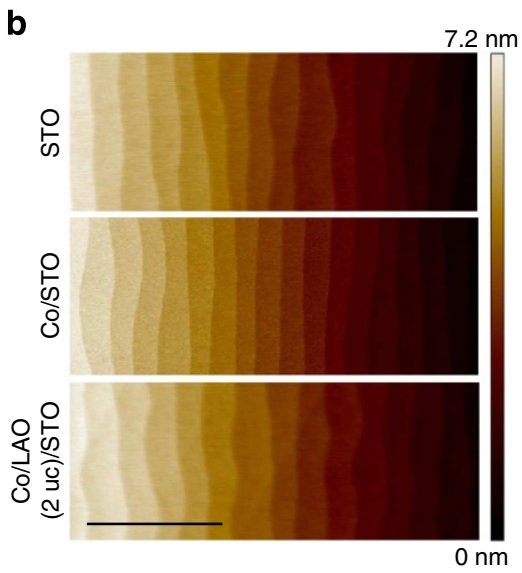

Figure 5 I In situ RHEED and ex situ AFM characterizations. (a) Monitoring of the RHEED (01) diffraction peak intensity versus time during the epitaxial growth of LAO films of various thicknesses (1-4 uc) on STO substrates. Inset: typical RHEED pattern of (left) an STO substrate, and (right) a 4-uc LAO thin film on STO, taken at $730^{\circ} \mathrm{C}$ in an oxygen partial pressure of $2.0 \times 10^{-4}$ mbar. Arrows indicate the end of the growth. Asterisks designate samples later covered with a $\mathrm{Co} / \mathrm{AlO}_{\mathrm{x}}$ capping. (b) AFM topography of a $\mathrm{TiO}_{2}$-terminated STO substrate and of Co/STO and $\mathrm{Co} / \mathrm{LAO}(2 \mathrm{uc}) / \mathrm{STO}$ samples. The $2-\mathrm{nm}$ Co thin film reproduces the steps and terraces of the terminated substrate. Scale bar, $1 \mu \mathrm{m}$. 
$\mathrm{LAO} / \mathrm{STO}$ interface as proposed in the polar catastrophe model $^{17,37}$, which suggests that cationic intermixing across the interface ${ }^{38}$ or oxygen vacancies ${ }^{39-41}$ only play a marginal role.

For spintronics and tunnelling spectroscopy applications, it is necessary to control the tunnel barrier resistance, which is usually achieved by tuning its thickness. In the $\mathrm{LaAlO}_{3} / \mathrm{SrTiO}_{3}$ case, a stringent limitation for efficient tunnelling was the existence of a minimum thickness of $4 \mathrm{uc}$ of $\mathrm{LaAlO}_{3}$ for the onset of conductivity. Our findings should therefore considerably facilitate spin injection ${ }^{42,43}$ or tunnel spectroscopy studies ${ }^{4-46}$ only achievable in perpendicular geometries, thereby paving the way toward $\mathrm{LaAlO}_{3} / \mathrm{SrTiO}_{3}$-based electronic and spintronic tunnelling devices with tunable tunnel resistances.

\section{Methods}

Samples fabrication. The $\mathrm{LaAlO}_{3}$ (LAO) films were grown by PLD on $5 \times 5 \mathrm{~mm}$ $\mathrm{TiO}_{2}$-terminated (001)-oriented $\mathrm{SrTiO}_{3}$ (STO) substrates. A single-crystal LAO target was ablated by a $\mathrm{KrF}(248 \mathrm{~nm})$ excimer laser at a repetition rate of $1 \mathrm{~Hz}$ and with a fluence of $\sim 1 \mathrm{~J} \mathrm{~cm}^{-2}$. The LAO deposition was performed in an oxygen partial pressure of $2.0 \times 10^{-4} \mathrm{mbar}$ and at a substrate temperature of $730^{\circ} \mathrm{C}$. Substrate-to-target distance was $63 \mathrm{~mm}$. The layer-by-layer growth mode allowed us to precisely control the LAO thickness through real-time monitoring of reflection high-energy electron diffraction intensity oscillations (see Fig. 5a). The samples were then annealed for $30 \mathrm{~min}$ in about $400 \mathrm{mbar}$ of oxygen at $500^{\circ} \mathrm{C}$ (refs 39,40). Finally, the LAO/STO heterostructures were cooled at $25^{\circ} \mathrm{C} \mathrm{min}^{-1}$ and kept in the same oxygen pressure for $\sim 30$ to $60 \mathrm{~min}$. PLD growth was followed by in situ deposition of a metallic cobalt electrode by magnetron sputtering at room temperature in a pure Ar atmosphere of $4.5 \times 10^{-4} \mathrm{mbar}$, resulting in a clean $\mathrm{Co} / \mathrm{LAO}$ interface. The $2-2.5-\mathrm{nm}$ Co thin films were capped with a $3-\mathrm{nm} \mathrm{AlO}_{x}$ layer to prevent cobalt oxidation. For the Co/STO sample, the same procedure of thermal and pressure cycles as the one described above were applied to the $\mathrm{TiO}_{2}$-terminated STO substrate, skipping only the LAO deposition step, before the Co deposition.

AFM characterization. We performed an atomic force microscopy (AFM) topography study of uncapped 2-nm Co films grown on a $\mathrm{TiO}_{2}$-terminated STO substrate and on an LAO/STO sample (see Fig. 5b). The Co was left to oxidize in air, which we believe could only increase surface roughness compared with Co metallic films capped with $\mathrm{AlO}_{x}$. We estimate the root mean square surface roughness value $\left(R_{\mathrm{q}}\right)$ of the Co film by averaging over four $3 \times 3 \mu \mathrm{m}^{2}$ images from different locations across the sample. $R_{\mathrm{q}}=1.49 \AA$ and $1.33 \AA$ for Co films grown on the STO substrate and the $\mathrm{LAO}(2 \mathrm{uc}) / \mathrm{STO}$, respectively. For comparison, the surface roughness of a terminated STO substrate from the same batch is of $1.50 \AA$. The surface roughness values are in fact dominated by the vicinal atomic steps and terraces of the substrate: considering $3.9 \AA$ steps height leads to $R_{\mathrm{q}}=1.13 \AA$. The AFM characterization study supports the smoothness and continuity of the 2-nm Co layer and suggests full coverage of the STO or LAO surfaces by the Co thin films.

DFT calculations. DFT calculations were performed using the all-electron full-potential linearized augmented plane wave method, as implemented in the WIEN2k code ${ }^{34,35}$. For the exchange-correlation potential, we used the GGA ${ }^{36}$. The muffin-tin spheres of the atoms were taken as: $2.3,1.8$ and 1.6 a.u. for $\mathrm{La} / \mathrm{Sr}$, $\mathrm{Ti} / \mathrm{Co} / \mathrm{Al}$ and $\mathrm{O}$, respectively. The energy cutoff for the plane wave representation in the interstitial is $E_{\max }^{\mathrm{wf}}=19 \mathrm{Ry}$ for the wave functions. The spherical harmonics inside the muffin-tin spheres are expanded up to $l_{\max }=10$, while the plane wave expansion of the charge density was truncated at $G_{\max }=12$ a.u. ${ }^{-1}$, where a.u. stands for atomic units.. The integrals over the Brillouin zone are performed with $36 k$-points in the irreducible part of the Brillouin zone using the Monkhorst-Pack special $k$-points approach ${ }^{47}$. We have chosen a symmetric slab with LAO and Co layers on both sides of the 4.5 -uc-thick STO substrate and a vacuum region between the slab and its periodic images of at least $10 \AA$. The Co atoms are adsorbed on top of the oxygen ions at the surface of $\mathrm{TiO}_{2}$ or $\mathrm{AlO}_{2}$. The in-plane lattice parameter is set to the GGA equilibrium lattice constant of STO $\left(a_{\mathrm{STO}}=3.92 \AA\right)$, and the atomic positions are fully relaxed within tetragonal symmetry.

\section{References}

1. Hwang, H. Y. et al. Emergent phenomena at oxide interfaces. Nat. Mater. 11, 103-113 (2012).

2. Ohtomo, A. \& Hwang, H. Y. A high-mobility electron gas at the $\mathrm{LaAlO}_{3} / \mathrm{SrTiO}_{3}$ heterointerface. Nature 427, 423-426 (2004).

3. Thiel, S., Hammerl, G., Schmehl, A., Schneider, C. W. \& Mannhart, J. Tunable quasi-two-dimensional electron gases in oxide heterostructures. Science 313, 1942-1945 (2006).
4. Ben Shalom, M., Sachs, M., Rakhmilevitch, D., Palevski, A. \& Dagan, Y. Tuning spin-orbit coupling and superconductivity at the $\mathrm{SrTiO}_{3} / \mathrm{LaAlO}_{3}$ interface: a magnetotransport study. Phys. Rev. Lett. 104, 126802 (2010).

5. Caviglia, A. D. et al. Tunable Rashba spin-orbit interaction at oxide interfaces. Phys. Rev. Lett. 104, 126803 (2010).

6. Reyren, N. et al. Superconducting interfaces between insulating oxides. Science 317, 1196-1199 (2007).

7. Caviglia, A. D. et al. Electric field control of the $\mathrm{LaAlO}_{3} / \mathrm{SrTiO}_{3}$ interface ground state. Nature 456, 624-627 (2008).

8. Bell, C. et al. Dominant mobility modulation by the electric field effect at the $\mathrm{LaAlO}_{3} / \mathrm{SrTiO}_{3}$ interface. Phys. Rev. Lett. 103, 226802 (2009).

9. Brinkman, A. et al. Magnetic effects at the interface between non-magnetic oxides. Nat. Mater. 6, 493-496 (2007).

10. Ariando, Wang, X. et al. Electronic phase separation at the $\mathrm{LaAlO}_{3} / \mathrm{SrTiO}_{3}$ interface. Nat. Commun. 2, 188 (2011).

11. Bert, J. A. et al. Direct imaging of the coexistence of ferromagnetism and superconductivity at the $\mathrm{LaAlO}_{3} / \mathrm{SrTiO}_{3}$ interface. Nat. Phys. 7, 767-771 (2011).

12. Dikin, D. A. et al. Coexistence of superconductivity and ferromagnetism in two dimensions. Phys. Rev. Lett. 107, 056802 (2011).

13. Li, L., Richter, C., Mannhart, J. \& Ashoori, R. C. Coexistence of magnetic order and two-dimensional superconductivity at $\mathrm{LaAlO}_{3} / \mathrm{SrTiO}_{3}$ interfaces. Nat. Phys. 7, 762-766 (2011).

14. Cen, C., Thiel, S., Mannhart, J. \& Levy, J. Oxide nanoelectronics on demand. Science 323, 1026-1030 (2009).

15. Li, L. et al. Very large capacitance enhancement in a two-dimensional electron system. Science 332, 825-828 (2011).

16. Förg, B., Richter, C. \& Mannhart, J. Field-effect devices utilizing $\mathrm{LaAlO}_{3}-\mathrm{SrTiO}_{3}$ interfaces. Appl. Phys. Lett. 100, 053506 (2012).

17. Nakagawa, N., Hwang, H. Y. \& Muller, D. A. Why some interfaces cannot be sharp. Nat. Mater. 5, 204-209 (2006).

18. Arras, R., Ruiz, V. G., Pickett, W. E. \& Pentcheva, R. Tuning the twodimensional electron gas at the $\mathrm{LaAlO}_{3} / \mathrm{SrTiO}_{3}(001)$ interface by metallic contacts. Phys. Rev. B 85, 125404 (2012).

19. van der Pauw, L. A method of measuring the resistivity and Hall coefficient on lamellae of arbitrary shape. Philips Tech. Rev. 20, 220-224 (1958).

20. Popovic', Z., Satpathy, S. \& Martin, R. Origin of the two-dimensional electron gas carrier density at the $\mathrm{LaAlO}_{3}$ on $\mathrm{SrTiO}_{3}$ Interface. Phys. Rev. Lett. 101, 256801 (2008).

21. Pentcheva, R. et al. Parallel electron-hole bilayer conductivity from electronic interface reconstruction. Phys. Rev. Lett. 104, 166804 (2010).

22. Joshua, A., Pecker, S., Ruhman, J., Altman, E. \& Ilani, S. A universal critical density underlying the physics of electrons at the $\mathrm{LaAlO}_{3} / \mathrm{SrTiO}_{3}$ interface. Nat Commun. 3, 1129 (2012).

23. Caviglia, A. D. et al. Two-dimensional quantum oscillations of the conductance at $\mathrm{LaAlO}_{3} / \mathrm{SrTiO}_{3}$ interfaces. Phys. Rev. Lett. 105, 236802 (2010).

24. Ben Shalom, M., Ron, A., Palevski, A. \& Dagan, Y. Shubnikov-De Haas oscillations in $\mathrm{SrTiO}_{3} / \mathrm{LaAlO}_{3}$ Interface. Phys. Rev. Lett. 105, 206401 (2010).

25. Ohresser, P. et al. DEIMOS: a beamline dedicated to dichroism measurements in the 350-2500 eV energy range. Rev. Sci. Instrum. 85, 013106 (2014).

26. Cowan, R. D. The Theory of Atomic Structure and Spectra (University of California Press, 1981).

27. de Groot, F. M. F., Fuggle, J., Thole, B. \& Sawatzky, G. $\mathrm{L}_{2,3} \mathrm{x}$-ray-absorption edges of $\mathrm{d} 0$ compounds: $\mathrm{K}^{+}, \mathrm{Ca}^{2+}, \mathrm{Sc}^{3+}$, and $\mathrm{Ti}^{4+}$ in $\mathrm{O}_{\mathrm{h}}$ (octahedral) symmetry. Phys. Rev. B 41, 928-937 (1990).

28. Mattheiss, L. Energy bands for $\mathrm{KNiF}_{3}, \mathrm{SrTiO}_{3}, \mathrm{KMoO}_{3}$, and $\mathrm{KTaO}_{3}$. Phys. Rev. B 6, 4718-4740 (1972).

29. Stavitski, E. \& de Groot, F. M. F. The CTM4XAS program for EELS and XAS spectral shape analysis of transition metal L edges. Micron 41, 687 (2010).

30. Salluzzo, M. et al. Structural and electronic reconstructions at the $\mathrm{LaAlO}_{3} / \mathrm{SrTiO}_{3}$ interface. Adv. Mater. 25, 2333-2338 (2013).

31. Salluzzo, M. et al. Orbital reconstruction and the two-dimensional electron gas at the $\mathrm{LaAlO}_{3} / \mathrm{SrTiO}_{3}$ interface. Phys. Rev. Lett. 102, 166804 (2009).

32. Fitzsimmons, M. et al. Upper limit to magnetism in $\mathrm{LaAlO}_{3} / \mathrm{SrTiO}_{3}$ heterostructures. Phys. Rev. Lett. 107, 217201 (2011).

33. Salluzzo, M. et al. Origin of interface magnetism in $\mathrm{BiMnO}_{3} / \mathrm{SrTiO}_{3}$ and $\mathrm{LaAlO}_{3} / \mathrm{SrTiO}_{3}$ heterostructures. Phys. Rev. Lett. 111, 087204 (2013).

34. Schwarz, K. \& Blaha, P. Solid state calculations using wien $2 \mathrm{k}$. Comput. Mater. Sci. 28, 259-273 (2003).

35. Blaha, P., Schwarz, K., Madsen, G. K. H., Kvasnicka, D. \& Luitz, J. WIEN2k, An Augmented Plane Wave Plus Local Orbitals Program for Calculating Crystal Properties (Vienna University of Technology, 2001).

36. Perdew, J. P., Burke, K. \& Ernzerhof, M. Generalized gradient approximation made simple. Phys. Rev. Lett. 77, 3865-3868 (1996).

37. Reinle-Schmitt, M. L. et al. Tunable conductivity threshold at polar oxide interfaces. Nat. Commun. 3, 932 (2012). 
38. Willmott, P. et al. Structural basis for the conducting interface between $\mathrm{LaAlO}_{3}$ and $\mathrm{SrTiO}_{3}$. Phys. Rev. Lett. 99, 155502 (2007).

39. Cancellieri, C. et al. Influence of the growth conditions on the $\mathrm{LaAlO}_{3} / \mathrm{SrTiO}_{3}$ interface electronic properties. EPL (Europhys. Lett.) 91, 17004 (2010).

40. Basletic, M. et al. Mapping the spatial distribution of charge carriers in $\mathrm{LaAlO}_{3} / \mathrm{SrTiO}_{3}$ heterostructures. Nat. Mater. 7, 621-625 (2008)

41. Liu, Z. Q. et al. Origin of the two-dimensional electron gas at $\mathrm{LaAlO}_{3} / \mathrm{SrTiO}_{3}$ interfaces: the role of oxygen vacancies and electronic reconstruction. Phys. Rev. $X$ 3, 021010 (2013).

42. Fert, A. \& Jaffrès, H. Conditions for efficient spin injection from a ferromagnetic metal into a semiconductor. Phys. Rev. B 64, 184420 (2001).

43. Reyren, N. et al. Gate-controlled spin injection at $\mathrm{LaAlO}_{3} / \mathrm{SrTiO}_{3}$ interfaces. Phys. Rev. Lett. 108, 186802 (2012).

44. Breitschaft, M. et al. Two-dimensional electron liquid state at $\mathrm{LaAlO}_{3} / \mathrm{SrTiO}_{3}$ interfaces. Phys. Rev. B 81, 153414 (2010).

45. Ristic, Z. et al. Photodoping and in-gap interface states across the metalinsulator transition in $\mathrm{LaAlO}_{3} / \mathrm{SrTiO}_{3}$ heterostructures. Phys. Rev. B 86, 045127 (2012).

46. Richter, C. et al. Interface superconductor with gap behaviour like a high-temperature superconductor. Nature 502, 528-531 (2013).

47. Monkhorst, H. J. \& Pack, J. D. Special points for brillouin-zone integrations. Phys. Rev. B 13, 5188-5192 (1976).

\section{Acknowledgements}

We acknowledge fruitful discussions with C. Deranlot. We would like to thank E. Jacquet for his support with the PLD and sputtering systems. We thank D. Sando and L. Phillips for a careful reading of the manuscript. We acknowledge SOLEIL for provision of synchrotron radiation facilities (project no. 20111079). This work was partly supported by the European Research Council (ERC Advanced Grant FEMMES, No. 267579), the German Science Foundation through project C3 within SFB/TR80 and grant h0721 for computational time at the supercomputers of the Leibniz Rechnenzentrum, Garching.

\section{Author contributions}

A.B. and M.B. supervised the project. E.L. and N.R. fabricated the samples, performed and analysed the transport measurements. E.L., N.R., R.M., V.C., F.C., P.O. and A.B. performed synchrotron measurements. E.L., N.R., R.M. and P.O. analysed the synchrotron data. D.D. and R.P. performed the ab-initio calculations. All authors contributed to interpretation of the results. E.L., N.R., D.D. and R.P. wrote the manuscript with inputs from P.O. and M.B.

\section{Additional information}

Supplementary Information accompanies this paper at http://www.nature.com/ naturecommunications

Competing financial interests: The authors declare no competing financial interests.

Reprints and permission information is available online at http://npg.nature.com/ reprintsandpermissions/

How to cite this article: Lesne, E. et al. Suppression of the critical thickness threshold for conductivity at the $\mathrm{LaAlO}_{3} / \mathrm{SrTiO}_{3}$ interface. Nat. Commun. 5:4291 doi: 10.1038/ncomms5291 (2014). 